\title{
A vision-based monitoring system for very early automatic detection of forest fires
}

\author{
J. Fernández-Berni, R. Carmona-Galán \& L. Carranza-González \\ Institute of Microelectronics of Seville - Centro Nacional de \\ Microelectrónica, Consejo Superior de Investigaciones Científicas y \\ Universidad de Sevilla, Avda. Reina Mercedes s/n, 41012 Sevilla, Spain
}

\begin{abstract}
This paper describes a system capable of detecting smoke at the very beginning of a forest fire with a precise spatial resolution. The system is based on a wireless vision sensor network. Each sensor monitors a small area of vegetation by running on-site a tailored vision algorithm to detect the presence of smoke. This algorithm examines chromaticity changes and spatio-temporal patterns in the scene that are characteristic of the smoke dynamics at early stages of propagation. Processing takes place at the sensor nodes and, if that is the case, an alarm signal is transmitted through the network along with a reference to the location of the triggered zone - without requiring complex GIS systems. This method improves the spatial resolution on the surveilled area and reduces the rate of false alarms. An energy efficient implementation of the sensor/processor devices is crucial as it determines the autonomy of the network nodes. At this point, we have developed an ad hoc vision algorithm, adapted to the nature of the problem, to be integrated into a single-chip sensor/processor. As a first step to validate the feasibility of the system, we applied the algorithm to smoke sequences recorded with commercial cameras at real-world scenarios that simulate the working conditions of the network nodes. The results obtained point to a very high reliability and robustness in the detection process.

Keywords: fire monitoring systems, automatic early detection, wireless sensors networks, artificial vision algorithms.
\end{abstract}




\section{Introduction}

Fire constitutes one of the primary agents of forest degradation. Indeed, there are regions where it is the main cause of forest destruction, e.g. the countries of the Mediterranean basin [1]. Forest fires also cause loss of human and animal lives as well as enormous economic damage. In order to minimize the effects of a forest fire, two fundamental aspects must be taken into account. First of all, early detection, and second, knowing its exact location. Stopping the spread of a forest fire beyond 15 minutes following ignition is very difficult in most cases [2]. By knowing its exact location, a fast and efficient intervention on the part of fire fighting resources can be delivered.

Traditionally, forest fires have been detected by means of human surveillance at lookout towers strategically situated to watch a large area. However, the hard working conditions of the fire-watchers have led to the development of automatic vision-based detection systems [3]. Regarding temporal and spatial resolution during the detection process, automatic systems based on static cameras $[4,5]$ achieve the best performance. These systems rely on infrared and/or visual cameras to detect forest fires within time intervals of minutes and, helped by a geographic information system (GIS), with a high spatial resolution [6]. However, two important, and connected, problems currently exist: cost and false alarm rate. In [7], a cost analysis of a commercial system is realized. The main conclusion is that the additional costs associated with the deployment and maintenance of the base system are only compensated when the number of unmanned lookout towers is at least five, what is only reasonable in the case of very large extensions. Besides, this kind of system is subject to numerous false alarms coming from different sources.

Real-time forest monitoring by a wireless sensor network (WSN) [8,9] is proposed as an alternative, in principle, to the above mentioned vision-based systems. A set of low-cost low-power sensors is deployed throughout the region of interest. These sensors can measure temperature, humidity and barometric pressure among other magnitudes [10]. Moreover, processing and communication capabilities are enabled on each sensor. According to observations of the weather conditions coming from the sensors, these systems determine the probability of fire, though arson can not be predicted.

We present here the preliminary results for a combined approach that can be classified as a wireless multimedia sensor network [11]. We propose a system based on vision-enabled sensor nodes distributed across the forest. By means of a tailored vision algorithm, that can be implemented into a single-chip sensor/processor, the sensors will watch for smoke in much smaller vegetation areas than those surveyed by cameras in automatic vision-based systems. In this way, we aim at improving the reliability of the detection process as well as achieving very early and precise forest fire detection. Once the fire is detected, an alarm signal is transmitted through the network. The targeted single-chip implementation of the sensor/processor and the simplicity of the communications infrastructure, where only alarm signals instead of complete images are transmitted, anticipate a very competitive cost. 


\section{Description of the system}

Consider the deployment of vision sensors throughout a forest. The sensors will be placed on poles, communication towers, pylons, trees, etc. in order to survey a determined vegetation area. Each sensor of the network will run on-site a vision algorithm in order to detect smoke arising among the vegetation. When a sensor detects smoke, a warning message is sent to a control center by multihopping.

One of the critical aspects in the design of a system for visual detection of smoke is to effectively capture the spatio-temporal dynamics introduced in the scene by the appearance of smoke. Precisely, it is in this, in determining which changes in the scene are caused by smoke and which not, where automatic-surveillance systems fail and trigger an unbearable amount of false alarms. Consider the sketch of the system in Fig. 1 where the area watched by sensor $S_{i}$, the first one to see the smoke, is highlighted. This area, $A_{S_{i}}$, is a critical parameter in the design of the system. If it is too small, we will be loosing the granularity of the changes and, therefore, we will not be able to classify changes in the scene as provenient of different sources, as all of them will affect the whole image sensor, consisting in an array of photodetectos, globally. On the other side, if it is too large, many different sources can introduce changes in the scene that, in the distance, are undistinguishable from those caused by smoke. In other words, watching from a long distance few things can be said about what causes the changes, while watching from too close all pixels will change as one impeding the identification of any propagation pattern. There will be then a minimum and a maximum area to be surveilled by the sensors that will be related to the system setup. As an initial estimation, a maximum of $500 \mathrm{~m}^{2}$ is contemplated in the tests that we report in this paper. Comparing with the systems analysed in [6], designed to detect smoke within a radius of $10 \mathrm{Km}$, i.e. a surveilled area of $314 \mathrm{Km}^{2}$, a camera with half a millon times more pixels would be required to achieve the same spatial resolution as the distributed vision sensor network. This drastic reduction on the coverage area on the part of the individual sensors when compared to the cameras of automatic vision-based systems intends to increase the reliability of the system and improve the temporal and spatial resolution of the detection process.

The proposed system presents several advantages when compared to the current automatic camera-based systems:

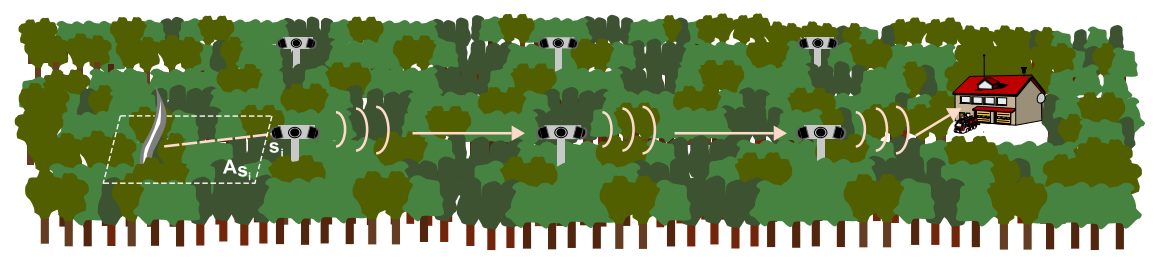

Figure 1: Sketch of the system. 
- Robustness: The failure of a sensor only affects to a very small fraction of the surveyed region. In a system based on cameras, the failure of a camera means that a large area stops being watched.

- Scalability: Regions of different sizes can be watched. The systems based on cameras are optimized to survey large areas.

- Reliability: The detection is not realized by analysing landscape images but small areas of vegetation. In this way, an adequate distribution and orientation of the sensors could avoid several sources of false alarms occuring in systems based on cameras, e.g. road dust or movements of clouds.

- Better temporal resolution: Due to the small size of the areas surveyed by the sensors, smoke can be detected within time intervals of seconds instead of minutes.

- Simpler smoke location: The exact location of the sensors is known in advance or at least by the time the network is deployed, therefore a geographical reference of the respective surveyed areas can be stored in each sensor. Due to the small size of the areas, this reference would suffice to rapidly locate the fire.

With respect to fire monitoring systems based on WSN, the crucial advantage is that vision sensors can detect all kind of forest fires, including arson.

Nonetheless, there are two primary research problems associated to the performance and cost of the proposed system: energy efficiency and reliability. The dense deployment and unattended nature of the vision sensors make it quite difficult to realize maintenance. Therefore, energy efficiency must be a major design goal of the system in order to prolong the lifetime of the batteries as much as possible. Regarding the reliability, consider an ideal system where only correct alarms are sent from the sensors and these alarms are always transmitted free from error until reaching the control center. In such a system, manual checking would not be necessary. This, in turn, means that the fire fighting resources could be deployed as soon as an alarm is received without any alarm confirmation delay. Therefore, the operation cost and response time of the proposed system greatly depend on the reliability of the detection. Both, energy efficiency and reliability of the system, depend on many different aspects. For the time being, we have focused on one element that has great influence on both: the vision algorithm. On one hand, each sensor must be continuously running it in order to detect smoke from the captured images. This constant processing represents the main source of power consumption at the sensors. A vision algorithm as simple as possible must be implemented in order to reduce the necessary computations and, therefore, the necessary energy, for smoke detection. On the other hand, the processing must be robust enough to prevent the generation and transmission of false alarms. These conditions imply that a trade-off between simplicity and robustness must be reached. algorithm. 


\section{Vision algorithm for smoke detection}

The algorithm hereby described is based on the analysis of the spatio-temporal patterns observed in the scene. Two elements will determine the sudden irruption of smoke in the field of view: a) changes in the chromaticity of the pixels in those areas now covered with smoke, with respect to their previous values, b) temporal and spatial correlation of these changes describing a dynamic behaviour that can only, in the better case, be associated with the appearance of smoke. Therefore, the algorithm runs a continuous capture loop in which a new image is sampled every $T_{C}$ seconds. These images are compared to a reference image, which constitutes the background, in order to detect changes introduced by smoke. This method of background subtraction [12], is suitable for motion detection in scenes with a relatively static background. In our case, the nature of the visual field of the sensors, basically composed of vegetation, entails that the background will very rarely undergo significant sudden changes. Instead, it will suffer gradual illumination changes throughout the day. Therefore, the reference image must be updated every time interval $T_{R}$. In order to synchronize the image rate and the updatings of the reference image, the relation between $T_{R}$ and $T_{C}$ must be:

$$
T_{R}=n T_{C} \quad\{n \in \mathbb{N}\}
$$

Note that the value of $T_{C}$ must reflect the temporal scale of the smoke dynamics in such a way that the image rate be just the necessary to detect smoke. In this way, the processing load and, consequently, the energy consumption, is greatly reduced.

Before carrying out the process of detection, the images must be binned, that is to say, divided into regions, or bins. Each bin is represented by the mean value of its pixels. It reduces the amount of data which must be analysed while still permitting the detection of smoke. Thus, the power consumption is adjusted to the real needs of the processing. In the proposed system, due to the small coverage area of the sensors, the physical dimensions of the different vegetation regions in the scene will be similar. Therefore, a simple regular binning can be implemented. In this case, all the bins are exactly equal. Their size, in pixels, can be defined as:

$$
S_{B}=W \times H
$$

The value of $S_{B}$ depends on the average variation in the number of smoke pixels during their appearance in the scene: the less the average variation, the smaller the necessary size of the bins to track the dynamics. Consider a sequence of $k$ consecutive images containing smoke captured by a sensor. The average variation in the number of smoke pixels during the sequence will be computed by:

$$
\bar{V}=\frac{\sum_{i=1}^{k}\left|P_{T}[i]-P_{T}[i-1]\right|}{k}
$$

where $P_{T}[i]$ is the total number of pixels affected by smoke in the $i$-th image of the sequence - suppose $P_{T}[0]=0$. According to this expression, the smoke dynamics 

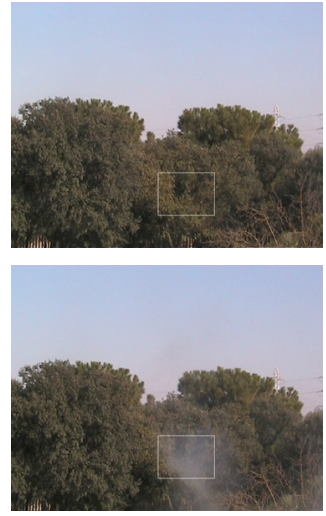

(a)
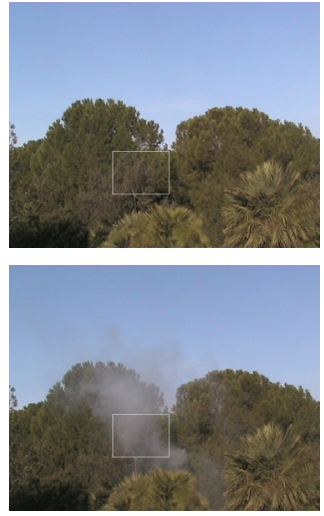

(b)
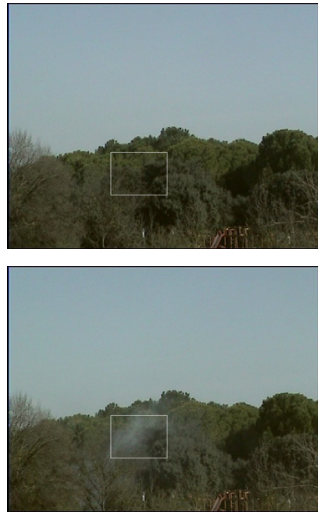

(c)

Figure 2: Three typical scenes with vegetation background without smoke and in presence of smoke.

Table 1: Normalized average increase, referred to the case without smoke, suffered by each component in the zones marked in Fig. 2 .

\begin{tabular}{|l|c|c|c|c|}
\hline & R component & G component & B component & Luminance \\
\hline Scene (a) & $13.8 \%$ & $14.5 \%$ & $20 \%$ & $14.9 \%$ \\
\hline Scene (b) & $12.5 \%$ & $13.4 \%$ & $19.5 \%$ & $13.8 \%$ \\
\hline Scene (c) & $11 \%$ & $12.8 \%$ & $16.4 \%$ & $12.7 \%$ \\
\hline
\end{tabular}

represented by these images can be correctly tracked whenever $S_{B}$ fulfills the following condition:

$$
S_{B} \leq \bar{V}
$$

Once the binning is realized, the first step of the algorithm is the detection of changes in the chromaticity of the bins. An option to discover smoke in any scene is the detection of the equalization of the R, G and B components in the pixels affected by smoke [13]. Nevertheless, for the detection of smoke arising among vegetation, we propose a more data-efficient option based only on the detection of sudden increases in the B component with respect to the background. The use of the B component is owing to its greater sensitivity to the changes generated by smoke in areas in which the vegetation is predominant, when compared to the $\mathrm{R}$ and $\mathrm{G}$ components and even to the combined luminance. Consider the scenes in Fig. 2. Here we have marked different zones where background is mainly constituted by vegetation. These frames correspond to different parts of the sequence, before and after a trace of smoke has appeared in the field of view. Table 1 shows the normalized average increase, referred to the image without smoke, undergone by each RGB component and luminance of the pixels within the 
marked zones in presence of smoke. It can be seen that, in all cases, the appearance of smoke among vegetation conveys a greater increase in the B component than that observed in the $\mathrm{R}$ and $\mathrm{G}$ components and the combined luminance.

Thus, defining $I_{B_{R}}(i, j)$ as the intensity of the B component at the bin $(i, j)$ of the reference image and $I_{B_{C}}(i, j)$ as the intensity of the B component at the bin $(i, j)$ of the image just captured, the condition to consider a foreground bin as a candidate bin to contain smoke is:

$$
I_{B_{C}}(i, j)-I_{B_{R}}(i, j)>\frac{p}{100}\left(I_{B_{M A X}}-I_{B_{M I N}}\right)
$$

where $p$ represents the threshold percentage of the $\mathrm{B}$ component signal range, set by $\left(I_{B_{M A X}}-I_{B_{M I N}}\right)$, that the B component of a foreground bin must rise with respect to the corresponding background bin in order to be labelled with a possible smoke condition. Once the candidate bins, that is, the bins fulfilling Eq. (5), are detected, the algorithm analyse them looking for spatio-temporal patterns which are characteristic of the smoke dynamics. This stage of the algorithm is divided into two phases: the detection and the confirmation phase. The detection phase starts when the first candidate bins are discovered, an instant denoted as $t_{0}$, and finishes at $t=t_{D}$. Then the confirmation phase is started, and will finish, if the result is positive, at time $t=t_{F}$, by sending an alarm message. The internal processing at both phases is described next.

First of all, in order to consider that smoke is present at the scene, a minimum number of candidate bins must exist. Let us define $N(t)$ as the number of candidate bins at time instant $t$. This parameter changes every $T_{C}$, that is, with every captured image. During the confirmation phase, the following expression must be fulfilled:

$$
N(t) \geq N_{M I N}\left\{t \in\left[t_{D}, t_{F}\right]\right\}
$$

where $N_{M I N}$ represents the minimum number of candidate bins to consider smoke. Below that, changes are associated to a different source and the confirmation phase is interrupted, returning to the pre-detection state.

Another important characteristic of the smoke dynamics is its gradual appearance into the scene. Once the first candidate bins are detected, new candidate bins must gradually appear until reaching at least $N_{M I N}$ at $t=t_{D}$. This fact can be described by means of two conditions. The first one is:

$$
t_{D}-t_{0} \leq T_{D_{M A X}}
$$

where $T_{D_{M A X}}$ represents the maximum time interval within which smoke must appear once the first candidate bins are detected. The second condition is:

$$
N(t)-N\left(t-T_{C}\right) \leq G_{M A X}\left\{t \in\left[t_{0}, t_{F}\right]\right\}
$$

where $G_{M A X}$ expresses the maximum permitted growth of candidate bins between two consecutive captured images during the smoke dynamics. The effect of not fulfilling Eq. (7) is the cancellation of the detection and the return to the predetection state. This is because the system considers that the source of the changes 
is too slow to behave as smoke, e.g. the shadow of the clouds. Failing to hold Eq. (8) means that the considered object grows much faster than smoke, therefore will come from a different source, e.g. a bird passing by. Finally, smoke does not appear as candidate bins scattered throughout the scene. On the contrary, it is formed by compact regions of candidate bins. Let us define $Z(t)$ as the number of 8-connected regions of candidate bins. Just like $N(t), Z(t)$ changes with every captured image. A compactness condition proper of smoke can be described as:

$$
Z(t) \leq Z_{\text {MAX }}\left\{t \in\left[t_{0}, t_{F}\right]\right\}
$$

being $Z_{M A X}$ the maximum permitted number of 8-connected regions during the smoke dynamics. In other words, failing to hold Eq. (9) means that a different and spread source is generating the changes, e.g. strong winds shaking the tree tops.

Therefore, the spatio-temporal dynamics of smoke can be summarised as follows: a minimum number of candidate bins $N_{M I N}$ must appear once the first candidate bins are discovered at $t=t_{0}$. The time instant at which $N_{M I N}$ is reached, $t=t_{D}$, establishes the end of the detection phase and the beginning of the confirmation phase. Besides, $t_{D}$ must fulfill Eq. (7), accounting in this way for the time scale of the gradual appearance of smoke in the scene. During the confirmation phase, whose duration is determined by $t_{F}-t_{D}$, the number of candidate bins must satisfy Eq. (6). Thus, we take into account that smoke does not disappear suddenly from the scene. Finally, Eq. (8) and Eq. (9) must be fulfilled during both the detection phase and the confirmation phase. The first one codifies the estimated growth rate of smoke during its propagation across the scene. The second accounts for its compactness characteristics.

\section{Preliminary tests}

In order to test the vision algorithm within the framework of the proposed system, we realized some video recordings at a natural environment. Approximately 80 minutes were recorded containing 16 sequences of gradual appearance of smoke following its natural evolution in scenes whose background is basically composed of vegetation. Commercial pyrotechnic smoke generators were used. Numerous sequences without smoke were also recorded to check the false alarm rate. As an example, the scenes in Fig. 2 correspond to three different tests. The range of vegetation areas covered by the cameras in the different tests was larger than $100 \mathrm{~m}^{2}$ and below $500 \mathrm{~m}^{2}$. Due to the orographical features of the scenario and the available means, it was not possible to record larger vegetation areas. Therefore, the parameters of the algorithm were adjusted to detect smoke within this range. In order to realize this parametrization, we analysed 9 of the 16 different sequences where smoke appears gradually [14]. The results are summarised in Table 2. With respect to $T_{R}$, longer recordings are mandatory to estimate an approximate value of this parameter. Even for the longest sequence recorded, whose duration is $458 \mathrm{~s}$, smoke detection was possible without updating the initial reference image.

Once the parameters were set, we applied the algorithm to all the recordings. Smoke was detected in the 16 sequences within a maximum interval of $24 \mathrm{~s}$ from 
Table 2: Parameters of the algorithm applied to the recordings.

\begin{tabular}{|c|c|c|c|c|c|c|c|}
\hline $\mathbf{T}_{\mathbf{C}}$ & $\mathbf{S}_{\mathbf{B}}$ & $\mathbf{p}$ & $\mathbf{N}_{\mathbf{M I N}}$ & $\mathbf{T}_{\mathbf{D}_{\text {MAX }}}$ & $\mathbf{G}_{\mathbf{M A X}}$ & $\mathbf{Z}_{\mathbf{M A X}}$ & $\mathbf{T}_{\mathbf{F}}$ \\
\hline $1 s$ & $15 \times 12$ & $10 \%$ & 14 & $20 s$ & 50 & 6 & $4 s$ \\
\hline
\end{tabular}
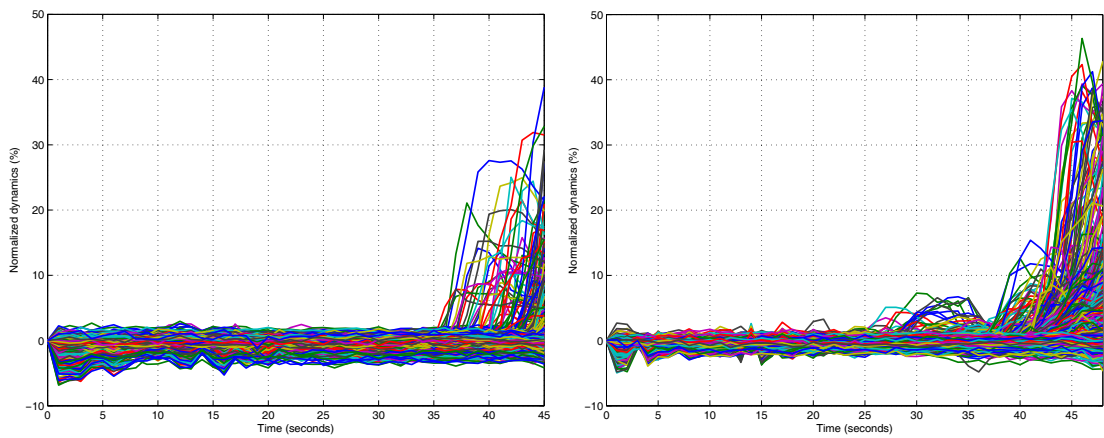

Figure 3: Normalized dynamics (\%) of all the bins with respect to the reference image in two recordings until smoke is detected.

its appearance in the scene. Besides, in the rest of the sequences, where smoke was not present, no false alarm was informed. Thus, the algorithm achieved a reliability of $100 \%$ for the conditions of the day of the recordings. No motion due to winds, nor changes introduced by birds, planes, people etc., triggered any false alarm. In Fig. 3, we represent the normalized dynamics of all the bins with respect to the reference image in two sequences until the time instant at which smoke is detected. The build-up of the detection and confirmation phases are pretty evident.

\section{Conclusions}

A forest fire detection system based on a wireless vision sensor network has been proposed. The system can detect forest fires within time intervals of seconds with a precise spatial location. One of the most important component, the vision algorithm, has been already defined. It looks for chromaticity changes and spatiotemporal patterns associated with the appearance of smoke in the scene. In order to validate the feasibility of the system, a set of recordings was carried out in realworld scenarios, simulation the working conditions that will be confronted by the real system. The results of applying the vision algorithm to these recordings point to a very high reliability and robustness in the process of detection. Our primary target now is the successfully implementation of the algorithm in a prototype sensor/processor chip that efficiently realizes detection at the nodes. 


\section{Acknowledgements}

This work is funded by Junta de Andalucía (CICE) through project 2006-TIC2352. The authors gratefully acknowledge the direction of the Parque del Alamillo, in Seville, for providing a natural but sufficiently controlled environment for the recordings, and the collaboration of the staff at Manuel Estalella S. L. (Barcelona) for their guidance in the selection of the pyrotechnics.

\section{References}

[1] FAO, Fire management - global assessment 2006. Technical report, Food and Agriculture Organization of the United Nations (FAO), 2007.

[2] INSA, FUEGO instrument design, prototype, construction and validation. Technical report, INSA Ingeniería y Servicios Aeroespaciales, 2000.

[3] San-Miguel-Ayanz, J., Ravail, N., Kelha, V. \& Ollero, A., Active fire detection for fire emergency management: Potential and limitations for the operational use of remote sensing. Natural Hazards, 35(3), pp. 361-376, 2005.

[4] Kührt, E., Knollenberg, J. \& Mertens, V., An automatic early warning system for forest fires. Annals of Burns and Fire Disasters, XIV(3), pp. 151-155, 2001.

[5] The Fire Watch Web Page. http://www.fire-watch.de.

[6] Schroeder, D., Evaluation of three wildfire smoke detection systems. Advantage Report, FERIC Forest Engineering Research Institute of Canada, 5(24), 2004.

[7] Schroeder, D., Operational trial of the forestwatch wildfire smoke detection system. Advantage Report, FERIC Forest Engineering Research Institute of Canada, 6(17), 2005.

[8] Yu, L., Wang, N. \& Meng, X., Real-time forest fire detection with wireless sensor networks. International Conference On Wireless Communications, Networking and Mobile Computing (WiMob'05), Montreal, Canada, pp. 1214-1217, 2005.

[9] Chaczko, Z. \& Ahmad, F., Wireless sensor network based system for fire endangered areas. Third International Conference on Information Technology and Applications (ICITA'05), Sidney, Australia, pp. 477-484, 2005.

[10] The Crossbow Inc. Web Page. http://www.xbow.com.

[11] Akyildiz, I., Melodia, T. \& Chowdhury, K., A survey on wireless multimedia sensor networks. Computer Networks, 51(4), pp. 921-960, 2007.

[12] Hu, W., Tan, T., Wang, L. \& Maybank, S., A survey on visual surveillance of object motion and behaviors. IEEE Transactions on Systems, Man and Cybernetics, 34(3), pp. 334-352, 2004.

[13] Chen, T., Yin, Y., Huang, S. \& Ye, Y., The smoke detection for early firealarming system based on video processing. IEEE International Conference on Intelligent Information Hiding and Multimedia Signal Processing (IIHMSP'06), California, USA, pp. 427-430, 2006.

[14] http://www.imse.cnm.es/ berni/. 